\title{
Desafios do Feedback na Avaliação Formativa, no Programa Interinstitucional de Interação Ensino-Serviço-Comunidade: Perspectiva de Alunos
}

\author{
Feedback Challenges in the Formative Assessment of the \\ Interinstitutional Program of Teaching-Service-Community \\ Interaction: the Students' Perspective
}

\author{
Guilherme Roberto Naves Miranda ${ }^{I}(1)$ \\ Tályta Freitas Pessoa ${ }^{I}$ (iD \\ Lilian Bianca Miller Martelo de Marco ${ }^{I}$ (iD \\ Arthur Carlos Bernardes Borges ${ }^{I}$ (DD \\ Breno Lima Silva Neves ${ }^{I}$ (iD \\ Igor Alves Miotto ${ }^{1}(\mathbb{D}$ \\ Matheus Alves Pedroso Ribeiro ${ }^{I}$ (D) \\ Luiza Engel Lorenzon ${ }^{I}$ (D) \\ Maira Vilagra Marques ${ }^{I}$ (iD
}

PALAVRAS-CHAVE

- Avaliação Educacional.

- Feedback.

- Educação Médica.
Introdução: Este trabalho aborda a utilização do mecanismo de feedback na avaliação formativa durante a formação médica, visto que esse instrumento é de grande importância para o estímulo dessa formação que se pauta pelas metodologias ativas de ensino. O estudo tem como objetivos caracterizar a aplicação dessa ferramenta e identificar possíveis falhas na utilização dela no cotidiano do módulo Pinesc, no curso de Medicina da Universidade Anhanguera (Uniderp). Método: Trata-se de uma pesquisa quantitativa e seccional que abrange alunos do primeiro ao oitavo semestre inseridos no módulo longitudinal Pinesc. Na coleta dos dados, utilizou-se um questionário com perguntas relativas à avaliação formativa que é realizada pelos preceptores, o qual é repassado aos acadêmicos de acordo com o cálculo de amostragem. O feedback é a atividade central da avaliação formativa e possibilita o desenvolvimento da capacidade reflexiva e autoavaliativa e de habilidades. Contudo, a utilização do feedback ainda encontra obstáculos principalmente pela dificuldade de os avaliadores elencarem os pontos negativos e as facilidades dos avaliados. Resultado: Neste estudo, foram relatadas algumas falhas e pontos positivos no processo de aplicação desse instrumento. Por conta disso, elencam-se e sugerem-se aperfeiçoamentos para uma melhor utilização do feedback e um aproveitamento acadêmico mais favorável. Conclusão: Como base da avaliação formativa, ofeedback ainda demonstra possuir muitas fragilidades quanto à sua forma e aplicação. 


\section{KEYWORDS}

- Educational Measurement

- Feedback.

- Medical Education.

Recebido em 5/12/19

Aceito em $2 / 8 / 20$

\section{INTRODUÇÃO}

Avaliar sempre foi um desafio. Segundo Sousa e Heinisch ${ }^{1}$, um método ideal de avaliação deve ter validade e fidedignidade e contribuir para que os estudantes desenvolvam mais suas competências, sendo capaz de configurar o rendimento escolar nas dimensões cognitiva, psicomotora e afetiva. De acordo com Borges et al. ${ }^{2}$, a ação de avaliar envolve múltiplas ferramentas interpretativas, como julgamentos e comparações, o que torna complexo o mecanismo de avaliação.

No âmbito da educação médica, a utilização da avaliação somativa como único método avaliativo vigente perdurou por um longo tempo. Conforme Gomes e Rego ${ }^{3}$, a base era um currículo tradicional que não estimulava adequadamente o desenvolvimento de autonomia, a capacidade de análise, o julgamento, a avaliação e o raciocínio crítico, investigativo e criativo. Com isso, na América Latina, a partir da década de 1970, intensificou-se o debate sobre a formação médica. Nesses debates, tanto as estruturas curriculares como o processo de ensino passaram a ser problematizados, procurando melhorar o nível de aprendizado e proporcionar um método resolutivo. Surgiu então o advento da metodologia ativa de aprendizagem, na qual o professor, além da função de ensinar, também assume o papel de facilitador da aquisição do conhecimento por parte do estudante.

As Diretrizes Curriculares Nacionais do Curso de Graduação em Medicina ${ }^{4}$ afirmam que este curso deve ter um projeto pedagógico centrado no aluno como sujeito da aprendizagem e no professor como facilitador e mediador, buscando uma formação integral e adequada. Nessa perspectiva, avaliações pontuais e classificatórias que procuram somente comparar o aluno a seus pares ao exigirem que ele atinja determinada pontuação preestabelecida vêm perdendo espaço no ambiente estudantil. Por sua vez, modelos que enfocam mais a trajetória percorrida pelo estudante para obter determinada habilidade e conhecimento e que procuram detectar em tempo hábil as possíveis dificuldades enfrentadas e ajudar a superá-las estão sendo moldes para a educação médica atual.

Assim, com base na compreensão da necessidade de uma avaliação processual com acompanhamento, diagnóstico e intervenções ao

\section{ABSTRACT}

Introduction: This present study assesses the use of the feedback mechanism in the formative assessment during medical training, considering this instrument is of great importance for this training stimulation, which is based on active teaching methodologies. The study aims to characterize the application of this tool and to identify possible failures in its application in the daily routine of the PINESC module in the Anhanguera University (Uniderp) medical course. Method: This is a quantitative, sectional research, including students from the first to the eighth semesters of the medical course, attending the PINESC longitudinal module. In the data collection, a questionnaire was used with questions related to the formative assessment performed by the preceptors, which is reported to the students according to the sample calculation. The feedback is the core activity of the formative assessment and allows the development of reflective and self-evaluation capacities and the development of skills. However, the use of feedback still faces barriers, mainly due to the difficulty of the evaluators in listing negative points and capabilities of the evaluated individuals. Result: In this study, some shortcomings and positive point use of feedback and thus a more favorable academic achievement. Conclusion: In conclusion, as the basis of formative evaluation, the feedback still shows many weaknesses regarding its form and application. 
Assim, em concordância com essas breves considerações, pretendese no presente trabalho discutir a avaliação formativa no contexto do Pinesc do curso de graduação em Medicina da Universidade Anhanguera Uniderp, enfatizando o papel do feedback e as considerações dos alunos acerca dele.

\section{METODOLOGIA}

Esta é uma pesquisa quantitativa, seccional, que abrange alunos do primeiro ao oitavo semestre (com exceção do sétimo, devido ao ingresso semestral de turmas na faculdade) inseridos no módulo longitudinal Pinesc, que possuem mais de 18 anos de idade e que estão regularmente matriculados do curso de Medicina da Universidade Anhanguera Uniderp, no município de Campo Grande, em Mato Grosso do Sul.

O instrumento de coleta de dados foi um questionário para os alunos, com perguntas sobre a avaliação formativa e o feedback, formulado após modificação do questionário de Marcos e Andrade ${ }^{7}$ e aplicado no dia 24 de novembro de 2018 na Universidade Anhanguera Uniderp, após aprovação do Comitê de Ética em Pesquisa sob o Certificado de Apresentação para Apreciação Ética (CAAE) n n 92606218.8.0000.5161, de acordo com a Resolução do Conselho Nacional de Saúde (CNS) nº 466/2012. O questionário era composto de 21 questões, sendo dividido em duas etapas. A etapa 1 consistia na caracterização do aluno e possuía como variáveis analisadas o semestre em curso do acadêmico, a quantidade de alunos por grupo de Pinesc e o número de vezes que ocorreu a troca de preceptor/UBSF (Unidade Básica Saúde da Família). Na segunda etapa, os questionamentos eram acerca da avaliação formativa, e as variáveis analisadas foram: a existência de um feedback do aluno para com o preceptor, o conceito de feedback, se era realizado ou não pelo preceptor e, se realizado, quais as características dele (periodicidade, individual ou em grupo, menção de pontos positivos e/ou negativos, coerência com o desempenho do acadêmico).

A quantidade de acadêmicos foi definida por uma amostra probabilística com base em parâmetros estipulados Fonseca e Martins ${ }^{8}$, para uma população finita com variáveis nominais ou ordinais. Após o cálculo, definiu-se uma amostra de 216 alunos, e realizou-se um cálculo simples de porcentagem para definir o número de questionários para cada semestre do curso.

Após a coleta, os dados obtidos foram salvos numa planilha do Excel. Posteriormente, importaram-se os dados, e efetuou-se a análise com o programa Epi Info ${ }^{\mathrm{m}}$. Foi utilizada uma análise estatística descritiva.

\section{RESULTADOS E DISCUSSÃO}

No âmbito da educação médica, para Gomes e Rego ${ }^{3}$, as metodologias ativas de ensino-aprendizagem - que têm como base o uso da avaliação formativa e dentro desta o feedback - possibilitam uma articulação entre a universidade, o serviço e a comunidade, já que criam possibilidades de interpretação e intervenção rápida sobre a realidade.

Anteriormente às análises feitas, uma das hipóteses levantadas neste trabalho era que havia um déficit na realização do feedback no âmbito do Pinesc. Se comprovada a hipótese, o resultado caracterizaria uma enorme deficiência na avaliação dos acadêmicos. Após a avaliação realizada pelo preceptor no Pinesc, todas as informações produzidas pela interação de professores e alunos para verificar o grau de aprendizado são explanadas em uma roda de discussões. Por meio do feedback dado pelo preceptor, os discentes são estimulados a refletir e expressar suas opiniões sobre as atividades do dia e a avaliação recebida.

Apesar do suposto na hipótese, 55,35\% (Gráfico 1) dos discentes entrevistados alegaram que o feedback era realizado contrariando a conjectura principal. Todavia, foi observada a existência de uma discrepância entre os semestres em curso pelos alunos quanto a esse dado, e nos semestres iniciais houve uma predominância da realização desse método de avaliação, em que 95\% (Gráfico 2) dos entrevistados do segundo semestre confirmaram que o feedback era realizado. Em contrapartida, no quinto semestre, $100 \%$ (Gráfico 2) relataram que o feedback não era realizado. Essa desconformidade entre os semestres pode ser relacionada ao que afirmam Poulos e Mahony' ${ }^{9}$ o impacto do feedback depende também do estágio da carreira universitária do avaliado. Os discentes que se encontram em estágios mais elevados (semestres finais) afirmaram que a significância do feedback não está relacionada apenas ao fornecimento de informações sobre como melhorar nota e desempenho, mas também ao que pode ser utilizado em sua prática profissional após a graduação.

Apesar disso, não se deve realizar o feedback somente para que a avaliação e a aprendizagem sejam de fato proveitosas e consoantes com o desempenho do aluno. Há características importantes que devem compor essa ferramenta para alcançar a efetividade. Uma característica importante desse tipo de avaliação é que o feedback deve ser utilizado como instrumento de retorno constante e contínuo, e não só em um

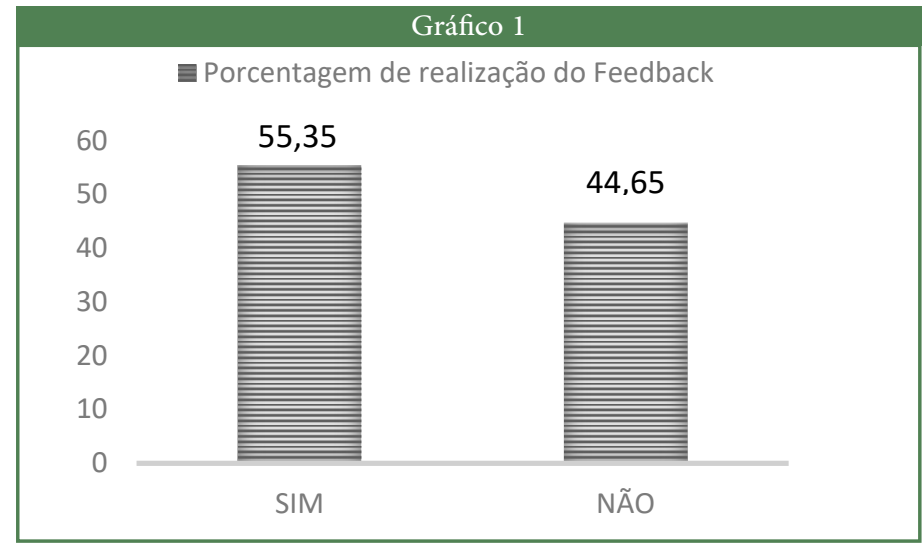

Fonte: Elaborado pelos autores

\section{Gráfico 2}

Porcentagem de realização do feedback de acordo com o semestre

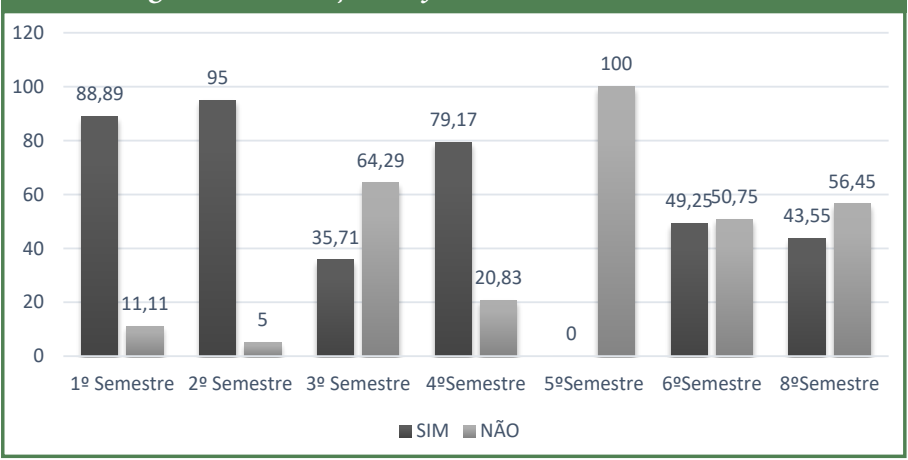

Fonte: Elaborado pelos autores

REVISTA BRASILEIRA DE EDUCAÇ̃̃o MÉDICA

3 44 (4) : e122; 2020 
momento privilegiado ${ }^{10}$. Para que essa autoavaliação seja possível, é recomendada certa regularidade na realização. Para Borges et al. ${ }^{2}$, as boas práticas em termos de avaliação formativa recomendam que o feedback seja feito regularmente, de modo a oferecer oportunidades para o estudante refletir e rever suas práticas ainda durante a experiência educacional. Pereira e Flores ${ }^{11}$ também discorrem sobre essa característica, afirmando que, se o feedback ocorre muito tempo depois da atividade desenvolvida, perde-se o sentido de ajudar na melhoria do desempenho daquele aluno, pois não terá relevância dentro do contexto de aprendizado daquela avaliação, atividade ou processo. Conquanto, nesta pesquisa, 54,62\% dos entrevistados afirmaram que ele era feito apenas ao final do semestre.

Outro aspecto é que $87,39 \%$ dos acadêmicos entrevistados afirmaram que os feedbacks não foram realizados de maneira individual, de modo que todos os integrantes do grupo estavam presentes. Para Borges et $\mathrm{al}^{2}$, o feedback deve ser dado de forma não constrangedora, de modo a proporcionar o entendimento e sanar possíveis dúvidas a respeito dos pontos elencados e da devolutiva feita. Além disso, o ambiente deve ser o mais acolhedor possível, para que o acadêmico se sinta estimulado a questionar e propor melhoras. Sendo assim, um feedback individual constituiria a melhor ferramenta de devolutiva, no qual o avaliador e o avaliado estabelecem um vínculo de confiança e respeito.

Além da necessidade de ser oferecido regularmente e de maneira individual, Zeferino et al. ${ }^{12}$ postulam que a efetividade é maior quando o feedback é de cunho assertivo, respeitoso, descritivo, oportuno e específico. Ou seja, a comunicação entre avaliador e avaliado precisa ser clara, objetiva e direta, avaliando os impactos e as consequências desse processo e propondo melhorias e mudanças. Professor e aluno também devem estar em consonância durante todo o processo, em que não deve haver julgamentos pessoais. Ademais, é importante que o preceptor aponte de forma clara as observações relatadas e especifique os pontos positivos e negativos. Referente ao conteúdo, conforme analisado, $81,36 \%$ dos entrevistados afirmaram que são elencados pontos positivos e negativos na realização do feedback. Associado a isso, 70,59\% dos alunos também afirmaram que essa ferramenta de avaliação é aplicada de maneira clara e objetiva pelos avaliadores.

Todavia, apesar de entenderem o conteúdo transmitido na avaliação, $49,30 \%$ dos entrevistados afirmaram que somente às vezes o feedback é coerente com o desempenho deles; além disso, 15,96\% afirmaram que nunca ou quase nunca consideram a avaliação coerente. Na definição de Bloom, Hastings e Madaus ${ }^{13}$, a avaliação formativa visa informar a localização das deficiências na organização do ensino para possibilitar correção e recuperação. Se não há coerência nesse feedback, podemos inferir que as deficiências não são bem determinadas, e, por conseguinte, a correção delas não acontece de forma ideal. Para Oliveira ${ }^{14}$, somente avaliações de qualidade, oportunas e orientadoras são auxiliares legítimas na construção do conhecimento em aspecto amplo, não apenas dos conteúdos propriamente ditos, como também de posturas e atitudes. Outro ponto que deve ser abordado refere-se ao fato de que, dentre os acadêmicos que assinalaram nunca ou quase nunca quanto à análise da conformidade da avaliação, $91,18 \%$ afirmaram que não expõem sua opinião sobre a incoerência do julgamento para o professor avaliador. Esse feedback inverso, que parte do acadêmico para o avaliador, informa ao professor os efeitos reais de seu feedback, o que lhe permitirá regular a própria ação com base nisso. Quando esse redirecionamento não ocorre, os erros cometidos pelo professor avaliador persistem, levando a uma deficiência em uma das funções da avaliação. Para Savaris ${ }^{15}$, essa função é a de clarificar e auxiliar no caminho da aprendizagem por meio de conceitos como: corrigir, ponderar, guiar e objetivar os estudos dos alunos.

Em um estudo realizado por Pereira e Flores ${ }^{11}$, em que o objetivo principal era o conhecer as perspectivas dos estudantes universitários sobre a avaliação no ensino superior, em particular sobre os métodos utilizados e o feedback, os resultados apontaram que os participantes consideram o feedback um elemento importante para a sua aprendizagem e valorizam as informações transmitidas pelos preceptores quando a sua aprendizagem depende delas. Nessa pesquisa, na concepção de 34,91\% dos acadêmicos, somente às vezes o feedback contribui para o aperfeiçoamento de habilidades e da formação estudantil. Em compensação, 19,81\% acreditam que essa ferramenta contribui sempre.

Os resultados demonstram que as percepções dos avaliados são, de modo geral, positivas. Porém, é perceptível que há várias deficiências quanto ao modo de realizar o feedback, e isso pode prejudicar o desenvolvimento de um pensamento crítico e impossibilitar melhorias no desempenho acadêmico. Para Daros e Prado ${ }^{16}$, o interesse do aluno em participar dessas etapas é fundamental; caso contrário, todo o fundamento do feedback não terá sentido, tornando o processo de avaliação formativa semelhante ao sistema de avaliação cognitiva, retirando assim seu objetivo maior que é enxergar o aluno em sua totalidade.

\section{CONCLUSÃO}

Os resultados deste estudo demonstram que os métodos de avaliação adotados pelos preceptores são de extrema importância e podem influenciar o processo de aprendizagem de forma negativa ou positiva. As maiores fragilidades identificadas por meio deste trabalho são referentes à maneira de aplicação do feedback, principalmente no que concerne ao tempo entre o término da atividade do acadêmico e o feedback transmitido pelo professor. Além disso, há uma deficiência quanto à coerência do feedback, à aplicação coletiva e ao feedback inverso, que parte do avaliado para o avaliador.

É necessário ressaltar que não existe um único jeito ou maneira de se transmitir um feedback, mas sim vários esquemas e/ou modelos que podem ser considerados aceitáveis. Conquanto, após análises, é possível considerar que um modelo de feedback efetivo é aquele que tem como atributos: ser claro, objetivo e coerente; ser realizado de forma individual, constante e contínua; ressaltar os pontos positivos do aluno e apontar suas deficiências. Ainda, possibilitar que o acadêmico reflita sobre a avaliação recebida e que também consiga realizar um retorno ao emissor do feedback sobre o resultado da ação quando este não lhe parecer justo. Além do mais, o avaliador deve estar sempre atento ao feedback que torne o aluno mais motivado e que ele entenda seu real desempenho.

Por fim, é preciso lembrar que o feedback fornecido necessita realmente instigar uma mudança do que está incorreto e o seguimento das boas práticas para que se alcance um melhor resultado no desempenho acadêmico e uma melhor relação ensino-serviço-comunidade.

\section{REFERÊNCIAS}

1. Sousa AP, Heinisch RH. Estudo sobre a avaliação aplicada no internato em clínica médica da Unisul. Rev Bras Educ Med. 2012;36(1):68-76.

2. Borges MC, Miranda $\mathrm{CH}$, Santana RC, Bollela VR. Avaliação

REVISTA BRASILEIRA DE EDUCAÇÃo MÉDICA

4 44 (4) : e122; 2020 
formativa e feedback como ferramenta de aprendizado na formação de profissionais da saúde. Revista da Faculdade de Medicina de Ribeirão Preto e do Hospital das Clínicas da FMRP. 2014;47(3):324-31.

3. Gomes AP, Rego S. Transformação da educação médica: é possível formar um novo médico a partir de mudanças no método de ensinoaprendizagem? Rev Bras Educ Med. 2011;35(4):557-66.

4. Brasil. Resolução CNE/CES no 3, de 20 de junho de 2014. Institui diretrizes curriculares nacionais do curso de graduação em medicina e dá outras providências. Diário Oficial da União; 6 jun. 2014; Seção 1.

5. Lima HLM, Picoli RP, Oliveira SLCB, Santos CC. Caderno de Prática Interdisciplinar de Interação Ensino, Serviço e Comunidade - Uniderp; 2018.

6. Ryan-Nicholls, KD. Preceptor recruitment and retention. Can Nurse. 2004;100(6):19-22.

7. Marco LBMM, Andrade SMO. Programa Interinstitucional de Interação Ensino-Serviço-Comunidade: desafios da avaliação na perspectiva de alunos e preceptores. Brasília. Tese [Mestrado em Saúde da Família]Fundação Oswaldo Cruz; 2019.

8. Fonseca JS, Martins GA. Curso de estatística. 6a ed. São Paulo: Atlas; 1996.

9. Poulos A, Mahony MJ. Effectiveness of feedback: the students' perspective. Assessment \& Evaluation in Higher Education. 2008;33(2):143-54.

10. Filatro A. Design de feedback e avaliação. São Paulo: Pearson; 2008.

11. Pereira DR, Flores MA. Avaliação e feedback no ensino superior: um estudio na Universidade do Minho. Revista Iberoamericana de Educación Superior. 2013;4(10):40-54.

12. Zeferino AMB, Domingues RCL, Amaral E. Feedback como estratégia de aprendizado no ensino médico. Rev Bras Educ Med. 2007;31(2):176-9.

13. Bloom BS, Hastings JT, Madaus GF. Evaluación del aprendizaje. Buenos Aires: Troquel; 1975.
14. Oliveira GP. Avaliação formativa nos cursos superiores: verificações qualitativas no processo de ensino aprendizagem e a autonomia dos educandos. Revista Iberoamericana de Educación. 2002. Disponível em:<https://rieoei.org/historico/deloslectores/261Pastre.PDF $>$. Acesso em 25 de março de 2019.

15. Savaris RF. Avaliação formativa entre alunos de medicina do quarto ano: relato de experiência. Rev Bras Educ Med. 2009;33(1):111-5.

16. Daros FAG, Prado MAM. Feedback no processo de avaliação da aprendizagem no ensino superior. XII Congresso Nacional de Educação, 2015 [acesso em 11 ago 2020]. Curitiba: Pontifícia Universidade Católica do Paraná; 2015. Disponível em: https:// educere.bruc.com.br/arquivo/pdf2015/17456_9283.pdf.

\section{CONTRIBUIÇÃO DOS AUTORES}

Arthur Carlos Bernardes Borges, Breno Lima Silva Neves, Guilherme Roberto Naves Miranda, Igor Alves Miotto, Maira Vilagra Marques, Matheus Alves Pedroso Ribeiro e Tályta Freitas Pessoa participaram da concepção do estudo, da coleta e análise de dados e da produção e escrita do artigo. Luiza Engel Lorenzon participou da confecção e escrita do artigo. Lilian Bianca Miller Martelo de Marco participou da concepção do estudo e da escrita e revisão do artigo.

\section{CONFLITO DE INTERESSES}

Os autores declaram não haver conflito de interesses neste estudo.

\section{ENDEREÇO PARA CORRESPONDÊNCIA}

Guilherme Roberto Naves Miranda. Rua Epitácio Pessoa, 681, Vila Vilas Boas, Campo Grande, MS, Brasil. CEP: 79051-090.

E-mail: guilhermernm9704@hotmail.com 